\title{
Fibrés semi-positifs et semi-négatifs sur une variété Kählérienne compacte.
}

\author{
JUAN GIRBAU (Barcelona, España) (*)
}

Summary. - Let $M$ be a kaehlerian compact manifold and $E \stackrel{r}{\rightarrow} M$ a holomorphic line bundle. In the first part two vanishing theorems for $H^{p, 0}(E)$ and $H^{0 . q}(E)$ are obtained. In the second part the following theorems are proved: 1) Let $E \stackrel{\leftrightarrow}{\rightarrow}$ be a holomorphio vector bundle weakly semi-definite negative. If the Ricci tensor of $M$ is $\geqslant 0$ on $M$ and $>0$ in a point, then $H^{n, 0}(E)=0, p>0$, 2) Let $M^{\prime}$ be a kaehlerian manifold with negative bisectional holomorphic curvature. Let $M$ be a compact complex submanifold. There is no holomorphic vector field of $M^{\prime}$ over $M$ and there is no locally trivial strict deformation of $M$. Intuitively this means that among the complex submanifolds close to $M$ (if there is any) there exists one with a complex structure different from that of $M$.

\section{1. - Introduction.}

Un des buts de ce travail est d'étendre les méthodes employés par A. LICHNERowicz dans [10] aux formes et tenseurs à coefficients dans un fibré vectoriel analytique complexe. Les principaux résultats sont énoncés dans les théorèmes $\mathrm{A}, \mathrm{B}, \mathrm{C}, \mathrm{D}$ et $\mathrm{E}$ des $\S 6,8$ et 9 . La première partie du théorème $B$, dans le cas particulier où le rang de $\left(c_{\alpha \bar{\beta}}\right)$ est constamment égal à $n$, devient le bien connu théorème d'annulation de K. KoDaIra [7]. Le cas où le rang de $\left(c_{\alpha \beta}\right)$ est constant a été aussi déjà étudié par E. Vesentint [13]. Les formules $(3.6)$ et $(4.2)$ qui sont à la base de nos raisonnements, ont été établies d'abord par l'auteur [4] dans le cas où $E$ est un fibré en droites et puis par P. GaUduchon [3] dans le cas général.

Dans un but de simplicité, on a regroupé dans le $\S 2$ les notations, formules et rappels utilisés.

Je tiens à remercier M. André Lrohnerowtoz qui m'a fait de remarques et de suggestions qui ont été d'une grande utilité et qui m'a aidé conerètement dans la démonstration du théorème $B$.

\section{2. - Préliminaires.}

Soit $M$ une variété hermitienne. Soit $E \stackrel{\pi}{\rightarrow} M$ un fibré vectoriel analytique complexe. Soit $h$ une métrique hermitienne positive sur chaque fibre de $E$. Dans une carte locale $U$ telle que $E \mid U$ est trivial, soit $\left\{s_{A}\right\}_{A=1 \ldots m}$ un système de $m$ sections de $E \mid U, m$ étant la dimension de la fibre, tel que dans chaque $x \in U$ constitue une base de $\pi^{-1}(x)$. Désignons par $h_{A B}=h\left(s_{A}, s_{B}\right)$. Soit $\left(h^{A B}\right)$ la matrice inverse de $\left(h_{A B}\right)$. La

(*) Entrata in Redazione l'11 novembre 1972. 
forme matricielle de type $(1,0): \omega_{A}^{B}=\left(d^{\prime} h_{A O}\right) h^{C B}$, donne lieu à une connexion dans $E$. Une $r$-forme différentielle sur $M$ à coefficients dans $E$ est par définition une section $C^{\infty}$ du fibré $A^{r}{ }^{\circ} T(M)^{*} \otimes E$, où ${ }^{\circ} T(M) \rightarrow M$ est le fibré des vecteurs tangents complexes. Soit $\nabla$ la connexion sans torsion dans ${ }^{e} T(M)$ determinée par la métrique hermitienne de $M$. Nous définissons l'opérateur $d_{E}$ qui agit sur les $r$-formes à coefficients dans $E$, localement, de la façon suivante:

$$
d_{E}\left(\sigma \otimes s_{A}\right)=d \sigma \otimes s_{A}+(-1)^{r} \sigma \wedge \omega_{A}^{B} \otimes s_{B}
$$

où $\sigma$ est une $r$-forme ordinaire sur $U$. Nous définissons anssi une connexion $\underset{E}{\nabla}$ sur le fibré $\left(\otimes{ }^{r} T(\dot{M})^{*}\right) \otimes E$ par:

$$
\underset{E}{\nabla}\left(\alpha \otimes s_{A}\right)=\nabla \alpha \otimes s_{A}+\alpha \otimes \omega_{A}^{B} \otimes s_{B}
$$

où $\alpha$ est un $r$-tenseur covariant sur $U$. Les opérateurs $d_{k}$ et $\nabla$ ainsi définis se décomposent en ses parties de type $(1,0)$ et $(0,1): d_{E}=d_{E}^{\prime}+d_{E}^{\prime \prime}, \underset{E}{\nabla}=\underset{E}{\nabla}+\nabla_{E}^{\prime \prime}$.

Une forme $\varphi$ de type $(p, q)$ à coefficients dans $E$ s'exprimera sur $U$ :

$$
\varphi=\frac{1}{p ! q !} \varphi_{\lambda_{1} \ldots \lambda_{p} \bar{\tau}_{1} \ldots \bar{\tau}_{q}}^{A} d z^{\lambda_{1}} \wedge \ldots \wedge d z^{\lambda_{p}} \wedge d z^{\bar{z}_{1}} \wedge \ldots \wedge d z^{\bar{\tau}_{q}} \otimes s_{A}
$$

Si $\varphi$ et $\psi$ sont deux formes de type $(p, q)$ à coefficients dans $E$, nous considérerons le produit scalaire local:

$$
(\varphi, \psi)=\frac{1}{p ! q !} \varphi_{\lambda_{1} \ldots \lambda_{p} \bar{\tau}_{1} \ldots \bar{\tau}_{q}} \overline{\psi^{\overline{\lambda_{1}} \ldots \bar{\lambda}_{p} \tau_{1} \ldots \tau_{q}} h_{A B}}
$$

Si la variété $M$ est compacte, ce que nous supposerons désormais, nous définissons le produit scalaire global: $\langle\varphi, \psi\rangle=\int(\varphi, \psi) \eta$, $\eta$ étant l'élément de volume. On définit les opérareurs $\delta_{E}^{\prime}$ et $\delta_{E}^{\prime \prime}$ par:

$$
\begin{aligned}
& \delta_{E}^{\prime} \psi_{\tau_{1} \ldots \tau_{p} \bar{\mu}_{1} \ldots \bar{\mu}_{q}}^{B}=-\nabla_{E}^{\tau_{1}} \psi_{\tau_{1} \ldots \tau_{p} \bar{\mu}_{1} \ldots \bar{p}_{q}}^{B} \\
& \delta_{E}^{\prime \prime} \psi_{\tau_{1} \ldots \tau_{p} \bar{\mu}_{1} \ldots \bar{\mu}_{q}}^{B}=(-1)^{p+1} \nabla_{E}^{\nabla_{1}} \psi_{\tau_{1} \ldots \tau_{p} \bar{\mu}_{1} \ldots \bar{\mu}_{q}}^{B} .
\end{aligned}
$$

Ces opérateurs sont les aidjoints de $d_{E}^{\prime}$ et $d_{E}^{\prime \prime}$ respectivement, par rapport au produit scalaire $\langle$,$\rangle . Nous définissons les deux laplaciens \frac{\Delta^{\prime}}{r}$ et $\frac{\Delta^{\prime \prime}}{\text { par: }}$

$$
\begin{aligned}
& \triangle_{E}^{\prime}=2\left(d_{E}^{\prime} \delta_{E}^{\prime}+\delta_{E}^{\prime} d_{E}^{\prime}\right) \\
& \underset{E}{\triangle}=2\left(d_{E}^{\prime \prime} \delta_{E}^{\prime \prime}+\delta_{E}^{\prime \prime} d_{E}^{\prime \prime}\right)
\end{aligned}
$$

Nous désignerons par $H^{p, q}(E)$ l'espace des formes $\varphi$ de type $(p, q)$ à coefficients dans $E$ telles que $\triangle^{\prime \prime} \varphi=0$. Désignons par $\Omega$ la forme de courbure de la connexion de $E$, c'est-à-dire:

$$
\Omega_{A}^{B}=d \omega_{A}^{B}-\omega_{A}^{o} \wedge \omega_{C}^{B}
$$


On pent vérifier les identités suivantes:

$$
\begin{gathered}
\left(d_{E}^{\prime}\right)^{2}=\left(d_{E}^{\prime \prime}\right)^{2}=0 \\
d_{E}^{2}=d_{E}^{\prime} d_{E}^{\prime \prime}+d_{E}^{\prime \prime} d_{E}^{\prime}=e(\Omega)
\end{gathered}
$$

où $e(\Omega)$ indique "produit extérieur par. $\Omega$ ".

Supposons maintenant que la métrique $g$ de $M$ est kählérienne. Soit $F$ la forme de Kähler:

$$
F=\sqrt{-1} g_{\alpha \bar{\beta}} d z^{\alpha} \wedge d z^{\bar{\beta}}
$$

Considérons les opérateurs $L=e(F)$ "produit extérieur par $F$ " et $A=i(F)$ "contraction intérieur par $F$ \%. On vérifie les identités suivantes:

$$
\begin{aligned}
& L d_{B}^{\prime}=d_{E}^{\prime} L \\
& \Lambda d_{E}^{\prime \prime}-d_{E}^{\prime \prime} A=-\sqrt{-1} \delta_{E}^{\prime} \\
& \text { (2.12) } \quad L d_{E}^{\pi}=d_{E}^{\prime \prime} L \\
& I_{v} \delta_{E}^{\prime}-\delta_{E}^{\prime} L=\sqrt{-1} d_{E}^{u}
\end{aligned}
$$

et ses duales:

$$
\begin{aligned}
& \Lambda \delta_{B}^{\prime}=\delta_{E}^{\prime} \Lambda \\
& \Lambda \delta_{E}^{\prime \prime}=\delta_{E}^{\prime \prime} \Lambda
\end{aligned}
$$

$$
\begin{aligned}
& L \delta_{E}^{\prime \prime}-\delta_{E}^{\prime \prime} L=\sqrt{-1} d_{E}^{\prime} \\
& \Lambda d_{E}^{\prime}-d_{E}^{\prime} \Lambda=-\sqrt{-1} \delta_{E}^{\prime \prime}
\end{aligned}
$$

En utilisant $(2.13),(2.18)$ et $(2.10)$ on obtient facilement:

$$
\Delta_{B}^{\prime}-\Delta_{B}^{\prime \prime}=2 \sqrt{-1}(\Lambda e(\Omega)-e(\Omega) \Lambda)
$$

Rappelons l'identité de Ricci:

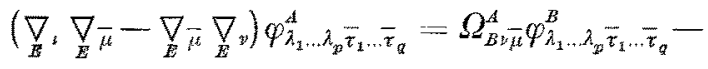

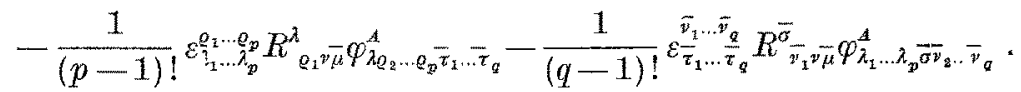

En faisant les calculs de $d_{E}^{\prime \prime} \delta_{E}^{\prime \prime}$ et de $\delta_{E}^{\prime \prime} d_{E}^{\prime \prime}$, en les additionant, et compte tenu de (2.20) on obtient l'expression suivante du laplacien $\triangle_{B}$ " agissant sur une forme $\varphi$ de type $(p, q)$ à coefficients dans $E$ :

$$
\triangle_{\vec{E}}^{\prime \prime} \varphi=-2 \underset{\nabla^{\sigma}}{\vec{\sigma}} \underset{E}{\nabla} \varphi+Q_{E}^{\prime \prime}(\varphi)+Q^{\prime \prime}(\varphi)+K(\varphi)
$$

$Q_{E}^{\prime \prime}, Q^{\prime \prime}$ et $K$ étant:

$$
\begin{aligned}
& Q_{E}^{\prime \prime}(\varphi)_{\lambda_{1} \ldots \lambda_{y} \bar{\tau}_{1} \ldots \bar{\tau}_{q}}^{A}=\frac{2}{(q-1) !} \varepsilon_{\bar{\tau}_{1} \ldots \ldots}^{\bar{\mu}_{1} \ldots \bar{u}_{q} q} \Omega_{B}^{A} \bar{\sigma}_{\bar{\mu}_{1}} \varphi_{\lambda_{1} \ldots \lambda_{p} \bar{\sigma} \bar{\mu}_{2} \ldots \bar{\mu}_{q}}^{B}, \\
& Q^{\prime \prime}(\varphi)_{\lambda_{1}, \lambda_{p} \bar{\tau}_{1} \ldots \bar{\tau}_{q}}^{A}=\frac{2}{(q-1) !} \varepsilon_{\bar{\tau}_{1} \ldots \bar{t}_{q}}^{\bar{\mu}_{1} \ldots \bar{\mu}_{q}} R_{\bar{\mu}_{1}}^{\bar{\sigma}} \varphi_{\lambda_{1} \ldots \lambda_{p}}^{A} \bar{\sigma}_{\mu_{2} \ldots \bar{\mu}_{q}},
\end{aligned}
$$

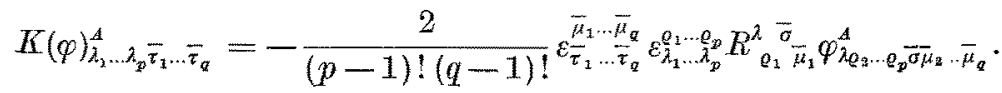

12 - Annali di Matematica 
Analoguement on obtient pour le laplacien $\frac{\Delta^{\prime}}{d}$ l'expression:

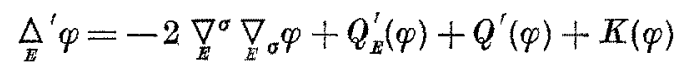

$Q_{E}^{\prime}$ et $Q^{\prime}$ étant:

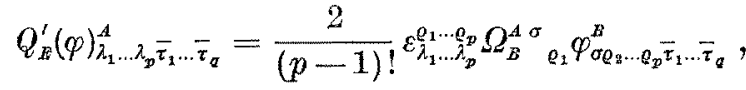

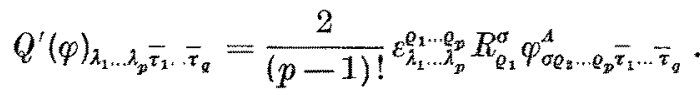

3. - Expressions de $\left\langle\triangle_{E}^{\prime} \varphi, \varphi\right\rangle$ et $\left\langle\triangle_{E}^{\prime \prime} \varphi, p\right\rangle$.

Appelons:

$$
\mathcal{S}^{\prime \prime}(\varphi)=Q_{z}^{\prime \prime}(\varphi)+Q^{\prime \prime}(\varphi)+K(\varphi)
$$

On aura:

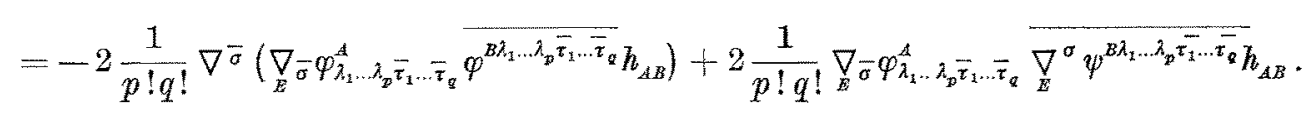

Le premier terme est une divergence et le deuxième est précisément égal à $2(p+1)$. $\cdot(q+1)\left(\nabla_{E}^{\prime \prime} \varphi, \nabla_{E}^{\prime \prime} \varphi\right)$.

Par intégration de (3.2) on obtient.

$$
\left\langle\triangle_{E}^{\prime \prime} \varphi, \varphi\right\rangle-\left\langle S^{\prime \prime}(\varphi), \varphi\right\rangle=2(p+1)(q+1)\left\langle\underset{Z}{\nabla^{\prime \prime}} \varphi, \underset{E}{\nabla^{\prime \prime}} \varphi\right\rangle
$$

Analoguement si nous appelons:

$$
S^{\prime}(\varphi)=Q_{E}^{\prime}(\varphi)+Q^{\prime}(\varphi)+K(\varphi)
$$

Nous aurons:

$$
\left\langle\triangle_{E}^{\prime} \varphi, \varphi\right\rangle-\left\langle S^{\prime}(\varphi), \varphi\right\rangle=2(p+1)(q+1)\left\langle\nabla_{E}^{\prime} \varphi, \underset{E}{\nabla^{\prime}} \varphi\right\rangle
$$

Par substitution dans $(3.5)$ de $\frac{\Delta^{\prime}}{\operatorname{par}}{\frac{\Delta^{\prime \prime}}{}}^{\prime}+2 \sqrt{-1}(\Lambda e(\Omega)-e(\Omega) \Lambda)$ on obitendra:

$$
\left\langle\Delta^{\prime \prime} \varphi, \varphi\right\rangle-\langle T(\varphi), \varphi\rangle=2(p+1)(q+1)\left\langle\underset{Z}{\nabla^{\prime}} \varphi, \nabla_{E}^{\prime} \varphi\right\rangle
$$

$T$ étant:

$$
T(\varphi)=2 \sqrt{-1}(e(\Omega) A-A e(\Omega)) \varphi+S^{\prime}(\varphi)
$$




\section{4. - Calcul local de l'opérateur $T$.}

Calculons d'abord la différence $e(\Omega) \Lambda-\Lambda e(\Omega)$. On aura:

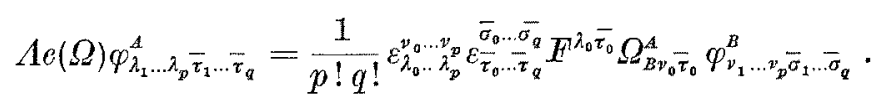

Ce terme se décompose en 4 , selon que $\lambda_{0}=v_{0}$ ou que $\lambda_{0}=v_{1}, \ldots, v_{p}$ et selon que $\bar{\tau}_{0}=\bar{\sigma}_{0}$ ou que $\bar{\tau}_{0}=\bar{\sigma}_{1}, \ldots, \bar{\sigma}_{q}$. On obtient ainsi:

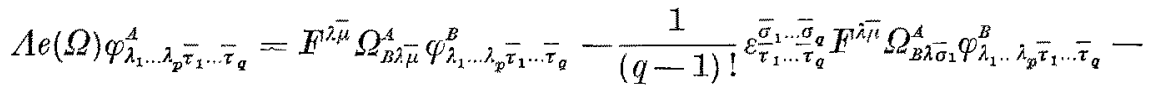

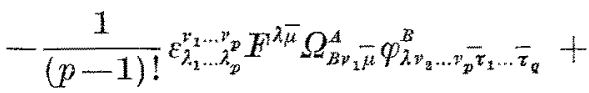

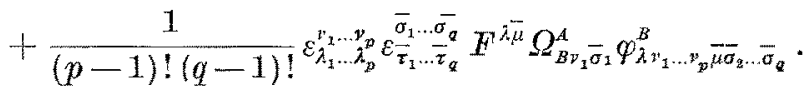

Mais ce dernier terme est précisément $e(\Omega) \Lambda \varphi_{\lambda_{1} \ldots \lambda_{p} \bar{\tau}_{1} \ldots \bar{\tau}_{a}}^{A}$ On $a: \sqrt{-1} F^{\lambda \bar{\mu}}=g^{\lambda \bar{\mu}}$. On aura done:

$$
\begin{aligned}
& 2 \sqrt{-1}(e(\Omega) A-\Lambda e(\Omega)) \varphi_{\lambda_{\mathrm{k}} . \lambda_{p} \bar{\tau}_{1} \ldots \bar{\tau}_{q}}^{A}= \\
& =-2 \Omega_{B \lambda}^{A \lambda} \varphi_{\lambda_{1} \ldots \lambda_{p} \bar{\tau}_{1}, \bar{z}_{q}}^{B}+Q_{E}^{A}(\varphi)_{\lambda_{1} \ldots \lambda_{p} \bar{\tau}_{1} \ldots \bar{\tau}_{q}}^{A}-Q_{E}^{\prime}(\varphi)_{\lambda_{1} \ldots \lambda_{p} \bar{\tau}_{2} \ldots \bar{\tau}_{q}}
\end{aligned}
$$

où $Q_{E}^{\prime}$ et $Q_{\bar{E}}^{\prime \prime}$ ont été définis par (2.22) et (2.26). On aura donc, avec notations evidentes:

$$
T(\varphi)=-2 \Omega_{B \lambda}^{A} \lambda^{\lambda} \varphi^{B}+Q_{E}^{\prime \prime}(\varphi)+Q^{\prime}(\varphi)+K(\varphi) .
$$

\section{5. - L'espace $T^{p}\left(E^{*}\right)$ des $p$-tenseurs holomorphes à coefficients dans $E^{*}$.}

Soit $E^{*}$ le fibré dual de $E$. Étant données $m$ sections $\left\{s_{A}\right\}$ de $E \mid U$ telles que dans chaque $x \in U$ constituent une base de $\pi^{-1}(x)$, considérons les sections duales $\left\{s^{\boldsymbol{A}}\right\}$ de $E^{*} \mid U$. Si $X$ est un tenseur contravariant antisymétrique de type $(p, 0)$ à coefficients dans $E$, nous désignerons par $\alpha(X)$ la forme de type $(p, 0)$ à coefficients dans $E$ donnée localement par:

$$
\alpha(X)_{\lambda_{1} \ldots \lambda_{p}}^{A}=h^{B A} \overline{\bar{X}_{A}^{Q_{1} \cdots \varrho_{p}} g_{\varrho_{1} \bar{\lambda}_{1} \ldots} g_{\varrho_{p} \bar{\lambda}_{q}}}=h^{B .4} \overline{X_{B} \bar{\lambda}_{1} \ldots \bar{\lambda}_{p}} .
$$

Désignons par $T^{p}\left(E^{*}\right)$ l'espace des tenseurs contravariants antisymétriques de type $(p, 0)$, holomorphes, à coefficients dans $E^{*}$. 
Proposition. - Si $X$ est un tenseur contravariant antisymétrique de type $(p, 0)$ à coefficients dans $E^{*}$, pour que $X$ soit de $T^{p}\left(E^{*}\right)$ il faut et il suffit que $\underset{E}{\nabla^{\prime}} \alpha(X)=0$.

Démostration. - Si $\varphi$ est une forme à coefficients dans $E$, donnée localement par: $\varphi=\varphi^{A} \otimes s_{A}$ où les $\varphi^{A}$ sont de formes ordinaires, de (2.2) et de la définition de $\omega$ on obient

$$
\nabla_{i i}^{\prime} \varphi^{A}=h^{C A} \nabla^{\prime}\left(h_{B \delta} \varphi^{B}\right)
$$

On a maintenant les équivalences suivantes:

$X$ holomorphe $\Leftrightarrow \partial_{\bar{\mu}} X_{B}^{\lambda_{1} \ldots \lambda_{p}}=0 \Leftrightarrow \nabla_{\bar{\mu}} X_{B}^{\lambda_{1} \ldots \lambda_{p}}=0 \Leftrightarrow \nabla_{\mu} \overline{X_{B}^{\lambda_{1} \ldots \lambda_{p}}}=0 \Leftrightarrow \nabla_{\mu} \overline{X_{B} \bar{\lambda}_{1}, \bar{\lambda}_{p}}=0 \Leftrightarrow$ $\Leftrightarrow \nabla_{\mu}\left(h_{B A} h^{C B} \overline{X_{C \bar{\lambda}_{1}} \ldots \bar{\lambda}_{p}}\right)=0 \Leftrightarrow \nabla_{\mu}\left(h_{B A} \alpha(X)_{\lambda_{1} \ldots \lambda_{p}}^{B}\right)=0$

et compte tenu de (5.1) cette dernière égalité est équivalente à:

$$
\nabla_{E}^{\prime} \alpha(X)=0
$$

\section{6. - Résultats pour les fibrés en droites.}

Soit $M$ une variété kählérienne compacte. Soit $E \stackrel{\pi}{\rightarrow} M$ un fibré holomorphe, en droites, muni d'une métrique hermitienne $h$. Soit $\Omega$ la forme de courbure de la connexion déterminée par cette métrique. Il est connu que $\Omega /(2 \pi \sqrt{-1})$ est un représentant de la première elasse de Chern de $E$. Réciproquement si $\Omega$ est une forme différentielle de type $(1,1)$ telle que $\Omega /(2 \pi \sqrt{-1}) \in c_{1}(E)$, il existe une métrique $h$ sur $E$ telle que $\Omega$ est la forme de courbure de la connexion déterminée par $h$. On dit que $c_{1}(E) \geqslant 0$ s'il existe $\Omega /(2 \pi \sqrt{-1}) \in c_{1}(E)$ tel que la forme hermitienne $\left(\Omega_{\alpha-\bar{\beta}}\right)$ est semidéfinie positive.

Nous appelerons tr $\Omega$ la trace de la forme hermitienne $\left(\Omega_{\alpha \bar{\beta}}\right)$, c'est-à-dire: tr $\Omega=\Omega_{\lambda}^{\lambda}$. Soit $g$ une métrique kählérienne sur $M$, et soit $R$ le tenseur de Ricci. Arec ces notations nous avons le théorème suivant:

THÉoRìme A. - 1) S'il existe $\Omega /(2 \pi \sqrt{-1}) \in e_{1}(E)$ tel que la forme hermitienne $e_{\alpha \bar{\beta}}=(-2 \operatorname{tr} \Omega) g_{\alpha \bar{\beta}}+p R_{\alpha \bar{\beta}}$ est semi-définie positive sur $M$, alors :

(a) Toute forme $\varphi \in H^{p, 0}(E)$ qui s'annulle dans un point est identiquement nulle.

(b) $H^{p, 0}(E)=0$ si $p>n-k, k$ étant le maximun du rang de la forme hermitienne $\left(c_{\alpha \bar{\beta}}\right)$ aux points de $M$.

(c) Pour $0<p \leqslant n-k$, on $a: \operatorname{dim}_{o} H^{p, 0}(E) \leqslant\left(\begin{array}{c}n-k \\ p\end{array}\right)$.

(d) Pour $p=0$ on a $H^{p, 0}(E)=0$ si tr $\Omega$ n'est pas identiquement nulle: 
2) S'il existe $\Omega /(2 \pi \sqrt{-1}) \in c_{1}(E)$ tel que la forme hermitienne $\left(c_{\alpha \bar{\beta}}\right)$ que nous avons introduite dans 1 ) est semi-définie négative sur $M$, alors:

(a $\left.a^{\prime}\right)$ Tout $X \in T^{p}\left(E^{*}\right)$ qui s'annulle dans un point est identiquement nul et $\operatorname{dim} T^{\prime o}(E) \leqslant \operatorname{dim} H^{p, 0}(E)$.

$\left(b^{\prime}\right) T^{p}\left(E^{*}\right)=0$ si $p>n-k$.

$\left(o^{\prime}\right)$ Pour $0<p \leqslant n-k$ on a: $\operatorname{dim}_{o} T^{p}\left(E^{*}\right) \leqslant\left(\begin{array}{c}n-k \\ p\end{array}\right)$.

$\left(d^{\prime}\right)$ Pour $p=0$ on a $T^{y}\left(E^{*}\right)=0$ si $\operatorname{tr} \Omega$ n'est pas identiquement nulle.

Ce théorème peut s'appliquer quand $R \geqslant 0$ avec tr $\Omega \leqslant 0$ et quand $R \leqslant 0$ avec tr $\Omega \geqslant 0$. Dans le cas où $E$ est le fibré trivial et le rang de $\left(c_{\alpha \bar{\beta}}\right)$ est constamment égal à $n$ ce théorème devient un bien connu résultat de $\mathrm{S}$. BOCHNER [1]

Démostration. - D'après $(4.2)$, sur les formes de type $(p, 0)$ l'opérateur $T$ sera: $T(\varphi)=-(2 \operatorname{tr} \Omega) \varphi+Q^{\prime}(\varphi)$. On aura:

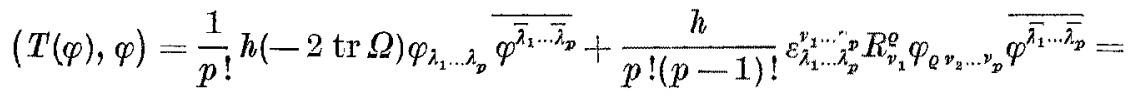

$$
\begin{aligned}
& =\frac{h}{p !}\left((-2 \operatorname{tr} \Omega) g_{\alpha \bar{\varrho}}+p R_{\alpha-\bar{\varrho}}\right) \varphi_{\lambda_{2} \ldots}^{\bar{Q} \ldots} \overline{p_{p}} \overline{\varphi^{\bar{\alpha} \bar{\lambda}_{2} \cdots \bar{\lambda}_{p}}} .
\end{aligned}
$$

Nous voyons done que l'opérateur $T$ a le même caractère que la forme hermitienne $c_{\alpha \bar{\beta}}=(-2 \operatorname{tr} \Omega) g_{\alpha \bar{\beta}}+p R_{\alpha \bar{\beta}}$. Supposons $e \geq 0$. Alors si $\varphi \in H^{p, 0}(E)$ nous aurons, d'aprés (3.6):

$$
-\langle T(\varphi), \varphi\rangle=2(p+1)\left\langle\underset{z}{\nabla^{\prime}} \varphi, \underset{\nabla^{\prime}}{\left.\nabla^{\prime} \varphi\right\rangle}\right.
$$

$T$ étant $\geqslant 0$, les deux membres doivent s'annuler. Soit $X=\alpha^{-1}(\varphi), \alpha$ étant l'opérateur défini dans $\$ 5$. Puisque $\nabla_{E}^{\prime} \alpha(X)=0, X$ est holomorphe. La fonction $(\varphi, \varphi)=$ $=i(X) \varphi$ sera alors holomorphe sur toute la variété, donc une constante. Si $\varphi$ s'annulle dans un point, doit done s'annuler sur toute la variété. Soit $x_{0}$ un point de $M$ dans lequel le rang de $\left(c_{\alpha \bar{\beta}}\right)$ est $k$. Soit $T_{x_{0}}^{\mathrm{L}, 0} \mathrm{l}^{\prime}$ 'espace des co-vecteurs de type $(1,0)$ dans le point $x_{0}$. Considérons l'application:

$$
\begin{aligned}
H^{p, 0}(E) & \rightarrow A^{p} T_{x_{0}}^{1,0} \\
\varphi & \rightarrow \varphi\left(\infty_{0}\right)
\end{aligned}
$$

qui est injective. L'image de cette application est contenue dans le sous-espace des formes $\varphi \in A^{p} T_{x_{0}}^{1,0}$ telles que:

$$
c_{\alpha \bar{Q}}\left(x_{0}\right) \varphi_{\lambda_{2} \ldots \lambda_{p}}^{\bar{Q}} \overline{\varphi^{\bar{\alpha} \bar{\lambda}_{2} \ldots \bar{\lambda}_{p}}}=0 .
$$


On voit en diagonalisant $\left(c_{\alpha \bar{\beta}}\right)$ par rapport à la métrique, dans ce point, que ce soussespace a dimension zéro si $p>n-k$ et dimension $\left(\begin{array}{c}n-k \\ p\end{array}\right)$ si $p \leqslant n-k$. La première partie du théorème est donc prouvée. Supposons maintenant $c \leqslant 0$. Si $X \in T^{p}\left(E^{*}\right)$ en vertu de la proposition du $\S 5, \underset{E}{\nabla^{\prime}} \alpha(x)=0$. On a alors, d'après $(3.6)$ :

$$
\left\langle\triangle^{\prime \prime} \alpha(x), \alpha(x)\right\rangle-\langle T(\alpha(x), \alpha(x))\rangle=0
$$

et puisque $T \leqslant 0$, les deux termes doivent s'annuler. On a done: $\triangle_{E}^{\prime \prime} \alpha(x)=0$, c'està-dire $\alpha(X) \in H^{v, 0}(E)$. La fonction $i(X) \alpha(X)$ sera holomorphe, done une constante. Si $X$ s'annulle dans un point, il doit être identiquement nul. L'application:

$$
T^{p}\left(E^{*}\right) \stackrel{\alpha}{\rightarrow} H^{p, 0}(E)
$$

étant injective, on aura: $\operatorname{dim} T^{y}\left(E^{*}\right) \leqslant \operatorname{dim} H^{p, 0}(E)$. L'application:

$$
\begin{aligned}
T^{v}\left(E^{*}\right) & \rightarrow A^{p} T_{x_{0}}^{1,0} \\
X & \rightarrow \alpha(X)\left(x_{0}\right)
\end{aligned}
$$

est aussi injective. Le reste de la démonstration esc maintenant trivial.

ThÉonÈme B. - Soit $K \rightarrow M$ le fibré canonique de $M$.

1) Supposons $c_{1}\left(E \otimes K^{*}\right) \geqslant 0$. Soit $c /(2 \pi \sqrt{-1}) \in c_{1}\left(E \otimes K^{*}\right)$ tel que la forme hermitienne $\left(c_{\alpha \bar{\beta}}\right)$ soit $\geqslant 0$. Soit $k$ le maximum du rang de $\left(c_{\alpha \bar{\beta}}\right)$ aux points de $M$. Alors $H^{p, 0}(E)=0$ pour $q>n-k$.

2) Si $\left(c_{\alpha \bar{\beta}}\right) \leqslant 0$, alors $\operatorname{dim} T^{q}(E) \leqslant \operatorname{dim} H^{0, q}(E)$ et $T^{q}(E)=0$ pour $q>n-k$.

En comparant cet énoncé avec celui du théorème $A$, on se pose les questions suivantes que nous n'avons pas su résoudre:

Avec les mémes hipothèses que dans 1 ) $d u$ th. $B$, est il encore vrai que toute $\varphi \in H^{0, q}(\mathbb{E})$ qui s'annulle dans un point est identiquement nulle? Est il encore vrai que $\operatorname{dim}_{o} H^{0, q}(E) \leqslant\left(\begin{array}{c}n-k \\ q\end{array}\right)$ quand $0<q \leqslant n-k$ ?

Quand $e_{1}(E)=0$ la répnose à ces questions est affirmative.

Démostration DU théorème. - D'après (3.1) l'opérateur $S^{\prime \prime}$ sur les formes de type $(0, q)$ sera: $s^{\prime \prime}(\varphi)=Q_{E}^{\prime \prime}(\varphi)+Q^{\prime \prime}(\varphi)$. On aura done:

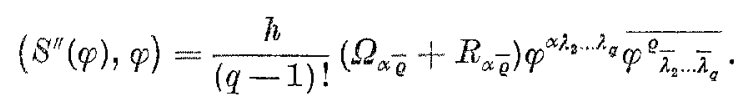

Nous voyons donc que l'opérateur $S^{\prime \prime}$ a le mêne caractère que la forme hermitienne $\left(\Omega_{\alpha \bar{\beta}}+R_{\alpha \bar{\beta}}\right)$. Mais $\Omega /(2 \pi \sqrt{-1})$ est un représentant quelconque de $c_{\lambda}(E)$ et $1 /(2 \pi \sqrt{-1})$ 
$R_{\alpha \bar{\beta}} d z^{\alpha} \wedge d z^{\bar{\beta}}$ est un représentant particulier de $e_{1}\left(K^{*}\right)$. La somme sera done un représentant quelconque de $c_{1}\left(E \otimes K^{*}\right)$. Nous pouvons poser $c_{\alpha \bar{\beta}}=\Omega_{\alpha \bar{\beta}}+R_{\alpha \bar{\beta}}$. Supposons d'abord $e \geq 0$. Soit $\varphi \in H^{\circ, q}(E)$. D'après (3.3) on aura:

$$
\left.\left.-\left\langle S^{\prime \prime}(\varphi), \varphi\right\rangle=2(q+1)\right\rangle \underset{z}{\nabla^{\prime \prime}} \varphi, \nabla_{E}^{\prime \prime} \varphi\right\rangle
$$

Puisque $0>0$ les deux termes doivent s'annuler. Nous avons donc:

$$
0=\nabla_{\bar{\sigma}} \varphi_{\overline{\mu_{1} \cdots \bar{\mu}_{q}}}=\nabla_{\bar{\sigma}} \varphi_{\bar{\mu}_{1} \ldots \bar{\mu}_{q}}
$$

Done:

$$
0=\nabla_{\bar{\sigma}} \varphi^{\mu_{1} \ldots \mu_{\alpha}}=\partial_{\bar{\sigma}} \varphi^{\mu_{\alpha} \ldots \mu_{\alpha}}
$$

Appelons $\beta$ l'opérateur qui consiste à monter les indices. $\beta$ applique une $(0, q)$-forme à coefficients dans $E$, dans un $(q, 0)$-tenseur à coefficients dans $E$. (6.5) et $(6.6)$ montrent que si $\varphi \in H^{0, q}(E), \beta(\varphi) \in T^{q}(E)$. Cela prouve que si $\varphi$ s'annulle dans un ouvert, doit s'annuler identiquement. Soit $x_{0}$ un point dans lequel le rang de $\left(c_{\alpha_{\beta}}\right)$ est $k$. Il existe un voisinage $U$ de $x_{0}$ sur lequel le rang est $k$. Pour $x \in U$ considérons l'application:

$$
\begin{aligned}
H^{0, q}(E) & \rightarrow \Lambda^{\alpha} T_{*}^{0,1} \\
\varphi & \rightarrow \varphi(x)
\end{aligned}
$$

L'image de cette application est contenue dans le sous-espace des formes $\varphi \in \Lambda^{a} T_{x}^{0,1}$ telles que

$$
c_{\alpha \bar{e}}(x) \varphi^{\alpha \lambda_{2} \ldots \lambda_{p}} \overline{\varphi^{Q_{\lambda_{2}} \ldots \lambda_{p}}}=0
$$

Ce sous-espace a dimension zéro si $q>n-k$. On a alors $\varphi(x)=0 \forall x \in U$, done $\varphi$ est identiquement nulle. La démonstration de la deuxième partie du théorème est analogue à celle du théorème $\mathrm{A}$.

On peut prouver (voir par exemple [7]) que:

$$
H^{0, q}(E) \cong H^{n, q}\left(E \otimes K^{*}\right) \cong H^{0, n-q}\left(E^{*} \otimes K\right) \text {. }
$$

En écrivant $E^{*}=E^{*} \otimes K \otimes K^{*}$ on obtient de ces isomorphismes et de 1) du théorème $B$ :

Théorìme $\mathrm{B}^{\prime}$. - S'il existe $c /(2 \pi \sqrt{-1}) \in c_{1}(E)$ tel que la forme hermitienne $\left(c_{\alpha \bar{\beta}}\right)$ est $\leqslant 0$, alors $H^{0, q}(E)=0$ pour $0 \leqslant q<k$, $k$ étant le maximum du rang de $\left(o_{\alpha \bar{\beta}}\right)$ aux point de $M$. 


\section{7. - Un exemple dû à E. Vesentini.}

(Pour cet exemple, voir [13]). Soient $M$ et $M^{\prime}$ deux variétés kählériennes compactes. Soit $f: M \rightarrow M^{\prime}$ une application holomorphe surjective telle que dans un point de $M$, au moins, l'application linéaire tangente soit aussi surjective. Soit $E^{\prime} \rightarrow M^{\prime}$ un fibré en droites, holomorphe. Soit $E \rightarrow M$ le fibré image inverse par $f$. Supposons que $o_{1}\left(E^{\prime}\right)<0$. Soit $c^{\prime} /(2 \pi \sqrt{-1}) \in c_{1}\left(E^{\prime}\right)$ tel que $\left(c_{\alpha \bar{\beta}}^{r}\right)<0$. Alors $f^{*}\left(c^{\prime}\right) /(2 \pi \sqrt{-1}) \in c_{1}(E)$ et on a $f^{*}\left(c^{\prime}\right) \leqslant 0$. Le maximum du rang de $f^{*}\left(c^{\prime}\right)$ aux points de $M$ est alors précisément égal à la dimension de $M^{\prime}$. Voici done une situation comme celle du théorème $B^{\prime}$.

\section{8. - Résultats pour les fibrés vectoriels quelconques.}

Soit $M$ une variété kählérienne compaete et $E \stackrel{\pi}{\rightarrow} M$ un fibré vectoriel analytique complexe. Soit $h$ une métrique hermitienne sur $E$. Nous définissons le tenseur de Nakano $H$ associé à $h$, de la façon suivante: $H(\varphi, X, \psi, Y)=h(\Omega(X, \bar{Y}) \varphi, \psi)$ où $X, Y$ sont de champs de vecteurs complexes sur $M$ et $\varphi, \psi$ sont de sections de $E$. ( $\Omega$ indique la courbure de la connexion induite dans $E$ par $h$ ). Le tenseur de Nakano vérifie: $H(\varphi, X, \psi, Y)=\overline{H(\psi, \overline{Y, \varphi, X})}$. On dit que $E$ est faiblement $\geqslant 0$ s'il existe une métrique $h$ dont le tenseur de Nakano $H$ vérifie $H(\varphi, X, \varphi X) \geqslant 0$, quels que soient $\varphi$ et $X$. Dans un système de coordonnées on aura:

$$
\begin{aligned}
& \varphi=\varphi \otimes s_{A} \quad X=X^{i} \frac{\partial}{\partial x^{i}} \quad i=1 \ldots 2 n, \\
& H(\varphi, X, \varphi, X)=H_{A i B j} \varphi^{A} X^{i} \frac{\varphi^{B} X^{j}}{} .
\end{aligned}
$$

On dit que $E$ est fortement $\geqslant 0$ s'il existe une métrique $h$ dont le tenseur de Nakano vérifie:

$$
H_{A i B j} X^{A i} \overline{X^{B j}} \geqslant 0
$$

quel que soit le 1-champ $X$ à coefficients dans $E$.

Dans les fibrés en droites les deux définitions coincident avec celle de $c_{1}(E) \geqslant 0$. La définition forte, due à NakAvo [12] est très utile pour l'obtention de théorèmes d'annulation (voir [12] et [5]), mais elle est peu géométrique. La définition faible, au contraire, est plus géométrique, mais on connajt moins de théorèmes d'annulation (voir [6]). Voici un résultat pour de fibrés faiblement semi-négatifs, qui est analogue au théorème $A$ :

Thtorìne C. - 1) Si le tenseur de Ricci $R$ de $M$ est $\geqslant 0$ sur $M$ et $>0$ dans un point, et si le fibré $E \stackrel{\pi}{\rightarrow} M$ est faiblement $\leqslant 0$, alors $H^{p, 0}(E)=0$ pour $p>0$. 
2) Si $R \leqslant 0$ sur $M$ et $<0$ dans un point, et si $E$ est faiblement $\geqslant 0$, alors $T^{\prime}\left(E^{*}\right)=0$ pour $p>0$.

DéMostratron. - Soit $\varphi$ une forme de type $(p, 0)$ à coefficients dans $E$. Sur une carte locale $U$, en fixant les indices $\lambda_{1}, \ldots, \lambda_{p}$ considérons la section de $E \mid U$ donnée par:

$$
\gamma_{\lambda_{1} \ldots \lambda_{p}}=\varphi_{\lambda_{1} \ldots \lambda_{p}}^{A} s_{A}
$$

On aura:

$$
H\left(\gamma_{\lambda_{1} \ldots \lambda_{p}}, \frac{\partial}{\partial z^{Q}}, \gamma_{\lambda_{1} \ldots \lambda_{p}}, \frac{\partial}{\partial z^{\varrho}}\right)=\varphi_{\lambda_{1} \ldots \lambda_{p}}^{B} \varphi_{\lambda_{1} \ldots \lambda_{p}}^{\sigma} H_{B_{Q} B Q}=\varphi_{\lambda_{1} \ldots \lambda_{p}}^{B} \overline{\varphi_{\lambda_{1} \ldots \lambda_{p}}^{G}} h_{\Delta C} \Omega_{B_{Q \bar{Q}}}^{A}
$$

Etant donné un point $\infty \in M$, prenons un système de coordonnées ortonormaux dans ce point et tel que la matrice $\left(R_{\alpha \bar{\beta}}\right)$ soit diagonale dans ce point. On aura dans ce point, compte tenu de (4.2):

$$
(T(\varphi), \varphi)=-\frac{1}{p !} 2 \sum_{\varrho \lambda_{1} \ldots \lambda_{p}} \Omega_{B \lambda}^{A}{ }^{\lambda} \varphi_{\lambda_{1} \ldots \lambda_{p}}^{B} \overline{\varphi_{\lambda_{1} \ldots \lambda_{p}}^{\sigma}} h_{A C}+\frac{2}{(p-1) !} \sum_{\varrho v_{z \rightarrow v_{p}}} R_{\varrho \varrho} \varphi_{\varrho \varrho}^{A} v_{2} \ldots \varrho_{p} \overline{\varphi_{\varrho}^{B} v_{2} \ldots v_{p}} h_{A B} .
$$

Mais le premier terme est positif d'après (8.3) puisque on suppose $E$ faiblement $\leqslant 0$, et le deuxième est aussi positif puisque on suppose $R \geqslant 0$ sur $M$ et $>0$ dans un point. Le théorème est maintenant trivial par le même raisonnement que dans le théorème $A$.

Soit maintenant $M$ une variété kählérienne compacte et $M^{\prime}$ une variété kählérienne quelconque. Soit $f: M \rightarrow M^{\prime}$ une application holomorphe. Soit $T^{1,0}\left(M^{\prime}\right)$ le fibré tangent do type $(1,0)$ de $M^{\prime}$. Soit $E$ l'image inverse de $T^{1,0}\left(M^{\prime}\right)$ par $f$. La métrique kählérienne de $M^{\prime}$ donnera lieu à une métrique $h$ sur E. L'opérateur $T$ s'écrira de la façon suivante sur les formes de type $(0,0)$ à coefficients dans $E$ :

$$
T(\varphi)=-2 \Omega_{B \varrho}^{A} e^{e} \varphi^{B}
$$

On aura done:

$$
(T(\varphi), \varphi)=-2 \Omega_{B Q}^{A}{ }^{Q} \varphi^{B} \overline{\varphi^{C}} h_{A Q}
$$

Un calcul simple montre que:

$$
\Omega_{B \varrho}^{A}{ }^{Q} \varphi^{B} \overline{\varphi^{\sigma}} h_{A \sigma}=R^{\prime}\left(\varphi, \bar{\varphi}, f_{*}\left(\frac{\partial}{\partial z^{\theta}}\right), f_{*}\left(\overline{\frac{\partial}{\partial z^{\mu}}}\right)\right) g^{\prime} \bar{\varphi}^{\bar{\mu}}
$$

où $R^{\prime}$ et $g^{\prime}$ sont respectivement la courbure et la métrique de $M^{\prime}$.

Supposons maintenant que $M$ est une sous-variété complexe de $M^{\prime}$ et que $f$ est l'inclusion canonique. Les formes de type $(0,0)$ à coefficients dans $E$ seront alors de champs de vecteurs de $M^{\prime}$ le long de $M$. De $(8.5),(8.6)$ et $(3.6)$ on obtient:

THÉonìme D. - Soit $M^{\prime}$ une variété kählérienne à courbure bissectionnelle holomorphe négative. Soit $M$ une sous-variété complexe compacte de $M^{\prime}$. Il n'existe 
pas de champs de $M^{\prime}$ holomorphes le long de $M$. (Un champ de $M^{\prime}$ holomorphe le long de $M$ est la donnée dans chaque $z \in M$ d'un vecteur $X_{z} \in T_{z}\left(M^{\prime}\right)^{1,0}$ dépendant holomorphiquement, de $z$ ).

Ce théorème suggère l'étude des déformations de $M$ par de sous-variétés de $\boldsymbol{M}^{\prime}$. Dans le § suivant on obtiendra un résultat concernant ces déformations.

\section{9. - Déformations d'une sous-variété compacte d'une variété kählérienne à courbure bissectionnelle holomorphe négative.}

Rappelons quelques définitions (voir [9]). Pour une famille différentiable $\mathscr{Y} \stackrel{\omega}{\rightarrow} X$ de structures complexes nous entendons la donnée d'un fibré différentiable $\vartheta \stackrel{\omega}{\longrightarrow} X$ sur une variété différentiable $X$, tel que:

(a) Chaque fibre $V_{t}=\omega^{-1}(t), t \in X$, est une variété analytique complexe dont la structure complexe est compatible avec la structure différentiable de $\vartheta$.

(b) Il existe un voisinage $U$ de chaque point de $\checkmark$ et un homéomorphisme différentiable $h$ de $\mathcal{U}$ dans $C^{n} \times \omega(\mathcal{U})$ tel que pour chaque $t \in \omega(\mathcal{W})$ la restriction $h_{t}$ de $h$ à $U \cap V_{t}$ est une application biholomorphe de $\mathcal{U} \cap V_{t}$ dans $C^{n} \times\{t\}$.

Pour une famille différentiable de sous-variétés d'une variété complexe $M^{\prime}$ nous entendons la donné d'une famille différentiable $\mathcal{V} \stackrel{\omega}{\rightarrow} X$ de structures complexes tel que chaque fibre est une sous-variété complexe de $\boldsymbol{M}^{\prime}$, et d'une application $\phi$ de $\mathcal{V}$ dans $\boldsymbol{M}^{\prime}$ dont la restriction à chaque fibre $\nabla_{t}$ est l'inclusion de $V_{i}$ dans $\boldsymbol{M}^{\prime}$. (Voir [9] pag. 386). Nous dirons qu'une telle famille ( $\stackrel{\oplus}{\rightarrow} X, \phi$ ) est une déformation par rapport à une fibre quelconque $V_{0}$. Nous dirons qu'une déformation $(\mathcal{H} \stackrel{\omega}{\rightarrow} X, \phi)$ est au sens stricte si pur chaque $t \in X$ et chaque voisinage $U$ de $t$ on a: $\phi\left(\omega^{-1}(U)\right) \neq \phi\left(V_{t}\right)$, l'inégalité comme sous-ensembles de $\boldsymbol{M}^{\prime}$.

THúfoRèME E. - Soit $M^{\prime}$ une variété kählérienne à courbure bissectionnelle holomorphe négative. Soit $M$ une sous-variété complexe, compacte de $M^{\prime}$. Il n'existe aucune déformation de $M$, au sens stricte, qui soit localement triviale.

Cet énoncé dit intuitivement que parmi les sous-variétés complexes proches à $M$, il y en a toujours, qui ont une structure complexe difiérente à celle de $\boldsymbol{M}$.

Démostration. - Repremnons les mêmes notations que dans [9] pag. 387. Nous avons les diagrames suivants:

$$
\begin{aligned}
& 0 \rightarrow \Theta \rightarrow \Pi \rightarrow T \rightarrow 0 \\
& \| \downarrow \downarrow \phi_{*} \downarrow \\
& 0 \rightarrow \Theta \rightarrow \Xi \rightarrow \Psi \rightarrow 0 \\
& \rightarrow T_{x} \stackrel{e}{\rightarrow} \mathfrak{H}^{1}(\Theta) \rightarrow \mathfrak{H}^{1}(I) \rightarrow \ldots \\
& \varrho_{d} \downarrow \quad \| \quad \downarrow \\
& \mathcal{H}^{0}(\Xi) \rightarrow \mathcal{H}^{0}(\Psi) \stackrel{\delta *}{\rightarrow} \operatorname{He}^{1}(\Theta) \rightarrow \mathcal{H}^{1}(\Xi) \rightarrow \ldots
\end{aligned}
$$


Puisque les conditions du théorème $D$ sont remplies nous aurons $\mathcal{H}^{\circ}(Z)=0$, e'est pourquoi $\varrho$ sera nulle si et seulement si $\varrho_{a}$ est nulle. Rappelons que $T_{X}$ est le faisceau qui a été construit en assignant à chaque ouvert $U$ de $X$ l'espace $T_{X}(U)$ des champs vectoriels différentiables sur $U$. Le faisceau $\mathfrak{H}^{\circ}(\Psi)$ a été construit en assignant à chaque $U$, l'espace $H_{0}(\mathcal{Y} \mid U, \Psi)$. L'homomorphisme $\varrho_{a}$ a été construit par passage au limite des homomorphismes:

$$
T_{z}(U) \stackrel{\mathscr{Q}_{d}(U)}{\rightarrow} H^{0}(\mathcal{V} / U, \Psi)
$$

où pour chaque $U, \varrho_{d}(U)$ a été construit de la façon suivante: Soit $\left\{\mathcal{U}_{i}\right\}$ un recouvrement de $\mathcal{Y} \mid U$ par ouverts $\mathcal{W}_{i}$ avec des homomorphismes de trivialisation associés $\mathcal{U}_{i}{ }^{{ }} \rightarrow C^{n} \times \omega\left(\mathcal{U}_{i}\right)$ d'accord avec la propriété $(b)$ de la définition de famille différentiable de structures complexes. Étant donné un champ $Y$ sur $U$ nous considérons un champ $Y_{i}$ sur chaque $\mathcal{U}_{i}$, projectable par $\omega$ sur $Y$, et dont la restriction à chaque fibre soit holomorphe. Sur $\mathcal{U}_{i} \cap \mathcal{U}_{j}$ la différence $Y_{i}-Y_{i}$ sera tangente aux fibres de $v$. Le système $\left\{Y_{i}\right\}$ définit une section de $T \mid \omega^{-1}(U)$. Désignons par $\pi_{1,0} \phi_{*}\left(Y_{i}\right)$ la partie de type $(1,0)$ de $\phi_{*}\left(Y_{i}\right) \cdot\left\{\pi_{1,0} \phi_{*}\left(Y_{i}\right)\right\}$ définira une section de $\Psi \mid \omega^{-1}(U) \cdot \varrho_{d}(U)$ applique $Y$ dans cette section. Le théorème se deduit maintenant du théorème 5.1 de [9] en observant que si $\varrho_{d}(U)$ est nulle on a $\phi\left(\omega^{-1}(U)\right) \subset \phi\left(V_{0}\right)$ quel que soit $\sigma \in U$, c'est-à-dire, la déformation n'est pas au sens stricte.

\section{BIBLIOGRAPHIE}

[1] S. Bochner, Tectors fields and Ricoi curvature, Bull. Amer. Math. Soc., 52 (1946), pp. $776-797$.

[2] S. S. CHeRs, Complex manifolds without potential theory, Van Nostrand, Princeton, 1967.

[3] P. Gadduchon, Sur les formes à valeurs dans un fibré vectoriel holomorphe, C. R. Acad. Sc. Paris, 273 (1971), pp. 398.401.

[4] J. GTRBAU, Sur les théorèmes d'annulation de Kodaira, C. R. Acad. Sc. Paris, 272 (1971), pp. $740-742$ et $273(1971)$, pp. 461-462.

[5] P. A. GRIfFiths, Hermitian differential geometry, J. Math. Mech., 14 (1965), pp. 117-140.

[6] P. A. Grimrins, Positive vectors bundles. Glabal analysis, Princeton Math., series n. 29 (1969), pp. 185-281.

[7] K. KODAIRA, On a differential geometrie method in the theory of anniytic stacks, Proc. N. Ac. Se. USA, 39 (1953), pp. 1268-1273.

[8] K. KoDarra, On cohomology grcups of compact analytio varieties, Proc. N. Ac. Sc. USA, 39 (1953), pp. 865-868.

[9] K. KodaIRA - D. C. SPEnCER, On dejormations on complex analytic structures, Ann. of Math., 63 (1958), pp. 328-466.

[10] A. LICHNERowicz, Variétés kählériennes et première classe de Chern, J. of Diff. Geom., 1 (1967), pp. 195-223.

[11] A. Lichnerowrcz, Applications harmoniques et variétes bählériennes, Inst. Naz. di Alta Math., Symposia Math. III, pp. 341-402.

[12] S. Nataxo, On complex analytic vector bundles, J. Math. Soc. Japan, 7 (1955), pp. 1-12.

[13] E. Vesenini, Osservazioni sulle strutture fibrate analitiche, Atti Ac. Naz. Lincei, 23 (1957), pp. $231-241$ et 24 (1957), pp. 505-512. 